\title{
Natural Sciences
}

Citation: Benavides-Serrato M, Contreras-Rueda YF, Barrios-Gardelis $\mathrm{LM}$, et al. Isolation and characterization of 17 polymorphic microsatellite loci for a sea urchin (Echinometra lucunter: Echinometridae). Rev. Acad. Colomb. Cienc. Ex. Fis. Nat. 44(172):759-767, julio-septiembre de 2020. doi: https:// doi.org/10.18257/raccefyn. 1113

Editor: Jaime Cantera

*Corresponding autor: Milena Benavides-Serrato; mbenavidess@unal.edu.co

Received: December 7, 2019

Accepted: March 31, 2020

Published: September 30, 2020

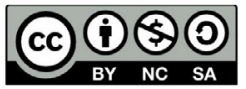

This is an open access article distributed under the terms of the Creative Commons Attribution License.
Original article

\section{Isolation and characterization of 17 polymorphic microsatellite loci for a sea urchin (Echinometra lucunter: Echinometridae)}

\author{
Aislamiento y caracterización de 17 microsatélites \\ polimórficos en un erizo de mar (Echinometra lucunter: \\ Echinometridae)
}

\author{
(1) Milena Benavides-Serrato ${ }^{1,2 *}$, (1) Yully Fernanda Contreras-Rueda ${ }^{1}$, \\ (1) Lina M. Barrios-Gardelis ${ }^{3}$, (1) Graeme Fox ${ }^{3}$, 나 Thomas D. Hughes ${ }^{3}$, \\ (1) Néstor Hernando Campos-Campos ${ }^{1}$, () Richard Preziosi ${ }^{3}$ \\ ${ }^{1}$ Universidad Nacional de Colombia, Sede Caribe - Instituto de Estudios en Ciencias del Mar-Cecimar, \\ El Rodadero, Santa Marta, Colombia \\ ${ }^{2}$ CEMarin - Corporation Center of Excellence in Marine Sciences, Bogotá, Colombia \\ ${ }^{3}$ Ecology and Environment Research Centre, Department of Natural Sciences, Manchester Metropolitan \\ University, Manchester, United Kingdom
}

\begin{abstract}
As a first step to establish the genetic structure of the sea urchin Echinometra lucunter lucunter throughout the Caribbean Sea, 26 microsatellite loci were isolated using Illumina paired-end sequencing, Next Generation Sequencing (NGS). We successfully optimized 17 loci for genotyping and the variation tested for 23 individuals from the Caribbean Sea and Tropical Eastern Atlantic Ocean. The allele number per locus (Na) ranged from four to 24 , the observed heterozygosity (Ho) from 0.682 to 1 , and the expected heterozygosity $(\mathrm{He})$ from 0.609 to 0.9304 . We detected no linkage disequilibrium between pairs of loci. These microsatellites will be used for the first time to detect the influence of marine barriers to genetic flow in the sea urchin E. lucunter lucunter throughout the Caribbean Sea. These new validated markers will be essential for current conservation and connectivity studies across the Caribbean Sea and the Atlantic Ocean.
\end{abstract}

Keywords: Marine connectivity; Echinodermata; Caribbean Sea; Microsatellites; Nuclear markers; Echinometra lucunter.

\section{Resumen}

Como primer paso para establecer la estructura genética del erizo de mar Echinometra lucunter lucunter a lo largo del Caribe, se aislaron 26 microsatélites usando secuenciación de extremo pareado (Next Generation Sequencing, NGS) Illumina. Se optimizaron exitosamente 17 marcadores y se probó su variación alélica en 23 individuos recolectados a lo largo del mar Caribe y en Cabo Verde, Atlántico oriental tropical. El número de alelos por locus $(\mathrm{Na})$ fluctuó entre cuatro y 24 , la heterocigosidad observada (Ho) entre 0,682 y 1 y la heterocigosidad esperada $(\mathrm{He})$, entre 0,609 y 0,9304 . No hubo desequilibrio de ligamento entre los pares de locus detectados. Los microsatelites aislados e identificados se usarán por primera vez para detectar la influencia de las barreras marinas en el flujo génico del erizo E. lucunter lucunter a lo largo del mar Caribe. Estos nuevos marcadores serán esenciales para la conservación y los estudios de conectividad a través del mar Caribe y el océano Atlántico, área donde se distribuye la especie.

Palabras clave: Conectividad marina; Echinodermata; Mar Caribe; Microsatelites; Marcadores nucleares; Echinometra lucunter. 


\section{Introduction}

Sea urchins play an ecologically important role in marine coastal ecosystems because of their high abundance and herbivorous feeding preference. Echinometra lucunter (Linnaeus, 1758) is often the dominant herbivore of benthic algae in shallow intertidal areas making it an excellent model to identify connectivity patterns for benthic organisms of the Atlantic Ocean.

E. lucunter is the most widely distributed species of the genus Echinometra. It has two subspecies, one in the Eastern Atlantic (Echinometra lucunter polypora Pawson, 1978) and the other in the Greater Caribbean (E. lucunter lucunter Linnaeus, 1758) which are separated from each other by 200000-250000 million years (McCartney, et al., 2000). The subspecies E. lucunter polypora was proposed by Pawson (1978) based on morphological differences found in specimens that live in the Central Atlantic islands of Ascension and St. Helena. Molecular analyses based on the CO1 gene (McCartney, et al., 2000) show that the closest approach is found between populations present in the African Atlantic, the Central Atlantic Islands (Ascension and St. Helena), and Brazil, suggesting that these populations belong to the subspecies E. lucunter polypora. The subspecies E. lucunter lucunter is present only in the Greater Caribbean.

Researchers have designed and used several types of molecular markers in the past to study genetic diversity within a species and analyse population distribution. Marker choice depends on the study, the particular species, and the questions to be answered (Sunnucks, 2000), therefore, it is necessary to use the correct molecular marker in line with the goals of the research and the available budget.

Microsatellites, or simple sequence repeats of loci (SSR), are tandem repeats of up to six nucleotides in the nuclear genome. The number of repeat units commonly ranges from around 5 to 40, with the number of repeats (and therefore sequence length) corresponding to different alleles (Selkoe \& Tonnen, 2006).

As a first step to establish the genetic structure and the influence of the marine barriers to the sea urchin Echinometra lucunter lucunter throughout the Caribbean Sea, we present the design, development, and characterization of 17 microsatellite markers in the sea urchin E. lucunter.

\section{Materials and methods}

\section{Development of microsatellites}

We selected eight $E$. lucunter lucunter individuals from three sites: Venezuela, Honduras, and Puerto Rico to develop microsatellite markers (Table 1).

Table 1. Samples used to design the simple sequence repeats (SSRs) of Echinometra lucunter

\begin{tabular}{|c|c|c|c|c|}
\hline Country & Coordinates & $\begin{array}{l}\text { Collection } \\
\text { date }\end{array}$ & $\begin{array}{l}\text { Sites/Number of } \\
\text { samples }\end{array}$ & $\begin{array}{l}\text { Approval organization } \\
\text { samples / approval number }\end{array}$ \\
\hline Venezuela & $\begin{array}{l}10^{\circ} 41^{\prime} 20.7^{\prime \prime} \mathrm{N} \\
63^{\circ} 52^{\prime} 29.11^{\prime \prime} \mathrm{W}\end{array}$ & $15 / 02 / 2016$ & Islote Lobos/2 & $\begin{array}{l}\text { Universidad Simón Bolivarl } \\
\text { by-product of the artisanal } \\
\text { fishery of the Araya Peninsula } \\
\text { in Venezuela }\end{array}$ \\
\hline Honduras & $\begin{array}{l}15^{\circ} 58^{\prime} 17.922^{\prime \prime} \mathrm{N} \\
86^{\circ} 28^{\prime} 14.36, \mathrm{~W}\end{array}$ & $14 / 05 / 2015$ & Cayos Cochinos/3 & $\begin{array}{l}\text { Instituto Nacional de } \\
\text { Conservación y Desarrollo } \\
\text { Forestal Areas Protegidas } \\
\text { y Vida Silvestre (ICF)/ } \\
\text { Resolution-DE-MP-080-2015 }\end{array}$ \\
\hline $\begin{array}{l}\text { Puerto } \\
\text { Rico }\end{array}$ & $\begin{array}{l}17^{\circ} 57^{\prime} 08.61^{\prime \prime} \mathrm{N} \\
67^{\circ} 033^{\prime} 24.30^{\prime \prime} \mathrm{W}\end{array}$ & $15 / 06 / 2016$ & Cayo Enrique/3 & $\begin{array}{l}\text { Departamento de Recursos } \\
\text { Naturales y Ambientales/ } \\
\text { DRNA: 2016-IC-052 }\end{array}$ \\
\hline
\end{tabular}


Genomic DNA was extracted from tube feet using the DNeasy Blood \& Tissue kit (Qiagen, Hilden, Germany) (Qiagen, 2006). The resulting DNA extractions were indexed and pooled to create a paired-end library using the Illumina Nextera ${ }^{\circledR}$ DNA Library Preparation Kit (Illumina, California, USA). Sequencing was carried out using an Illumina MiSeq platform (Illumina, California, USA) at the University of Salford, Salford, UK.

Microsatellite PCR markers were developed in-house using a previously published microsatellite design method (Fox, et al., 2019). The Griffiths, et al. (2016) workflow was used to detect microsatellites in the sequence data and design PCR primers. Multiindividual microsatellite identification (MiMi) (Fox, et al., 2019) was used subsequently as further quality control on potential markers.

To check for amplification success, we tested potential markers with the same eight DNA extractions used to design the microsatellites and extractions from additional individuals from the Colombian Caribbean Sea and the Tropical Eastern Atlantic Ocean. For the amplification steps we used the specifications of the Type-it ${ }^{\circledR}$ Microsatellite PCR kit (Qiagen, Hilden, Germany), (Qiagen, 2009) in a reaction volume of $5 \mu 1$ and the following thermal cycle: Step 1: $95^{\circ} \mathrm{C} / 5$ minutes; step 2: 28 cycles x $\left(95^{\circ} / 30\right.$ seconds for denaturation, $61^{\circ} \mathrm{C} / 90$ seconds for annealing, and $72^{\circ} \mathrm{C} / 30$ seconds for elongation); step 3: $60^{\circ} \mathrm{C} / 30$ minutes, and step 4: hold at $4^{\circ} \mathrm{C}$. We checked the final $5 \mu 1$ of PCR product solution on $1.8 \%$ agarose electrophoresis gel against a Hyperladder IV size standard (Bioline, London, UK) to confirm successful PCR amplification and the approximate size range of the fragments. Twenty-six SSR's were successfully visualized with these steps. To facilitate multiplexing, a universal tail PCR approach (Blacket, et al., 2012; Culley, et al., 2013) was used to add a fluorochrome to each of the markers (6FAM, TAMRA, HEX, and PET). Amplification success, thermal cycles, PCR features, and confirmation of successful gels were used as in the earlier step.

\section{Microsatellite screening}

In order to assess polymorphism of SSR's, we analysed 23 DNA samples from Cape Verde (Africa) and Caribbean localities (Table 2) using six multiplex (three-four markers each) and seven singleplex reactions (Table 3) using the PCR thermal cycles described previously. Confirmation of amplified SSR was again through visualizing on $1.8 \%$ agarose gels.

Table 2. Populations used to observe behaviour and polymorphism of microsatellites loci developed for Echinometra lucunter

\begin{tabular}{|c|c|c|c|c|}
\hline Country & Coordinates & $\begin{array}{l}\text { Collection } \\
\text { date }\end{array}$ & $\begin{array}{l}\text { Sites / number of } \\
\text { samples }\end{array}$ & Approval organization samples / approval number \\
\hline Honduras & $\begin{array}{l}15^{\circ} 58^{\prime} 17.92^{\prime \prime} \mathrm{N} \\
86^{\circ} 28^{\prime} 14.36^{\prime \prime} \mathrm{W}\end{array}$ & $14 / 05 / 2015$ & Cayo Cochinos $/ 3$ & $\begin{array}{l}\text { Instituto Nacional de Conservación y Desarrollo Forestal Áreas } \\
\text { Protegidas y Vida Silvestre (ICF)/ Resolution-DE-MP-080-2015 }\end{array}$ \\
\hline Puerto Rico & $\begin{array}{l}17^{\circ} 57^{\prime} 08.61 ’ \mathrm{~N} \\
67^{\circ} 03^{\prime} 24.30^{\prime \prime} \mathrm{W}\end{array}$ & $15 / 06 / 2016$ & Cayo Enrique/3 & $\begin{array}{l}\text { Departamento de Recursos Naturales y Ambientales/ DRNA: 2016- } \\
\text { IC-052 }\end{array}$ \\
\hline \multirow[t]{4}{*}{ Colombia } & $\begin{array}{l}12^{\circ} 27^{\prime} 3.6^{\prime \prime} \mathrm{N} \\
72^{\circ} 7^{\prime} 17.4^{\prime \prime} \mathrm{W}\end{array}$ & $01 / 07 / 2016$ & Cabo de la Vela/1 & \multirow{4}{*}{$\begin{array}{l}\text { Ministerio de Ambiente y Desarrollo Sostenible, República de } \\
\text { Colombia, Autoridad Nacional de Licencias Ambientales (ANLA): } \\
\text { Resolution number 255, } 12 \text { of March of } 2014 \\
\text { Export permit \#00848 }\end{array}$} \\
\hline & $\begin{array}{l}8^{\circ} 41^{\prime} 8.2 ” \mathrm{~N} \\
77^{\circ} 19^{\prime} 12.7^{\prime \prime} \mathrm{W}\end{array}$ & $15 / 06 / 2016$ & Capurganá/2 & \\
\hline & $\begin{array}{l}12^{\circ} 32^{\prime} 34^{\prime \prime} \mathrm{N} \\
81^{\circ} 44^{\prime} 45^{\prime \prime} \mathrm{W}\end{array}$ & $14 / 08 / 2016$ & San Andrés/3 & \\
\hline & $\begin{array}{l}14^{\circ} 17^{\prime} 4.52 ” \mathrm{~N} \\
80^{\circ} 22^{\prime} 55^{\prime \prime} \mathrm{W}\end{array}$ & $18 / 08 / 2016$ & Isla Cayo Serrana /1 & \\
\hline Venezuela & $\begin{array}{l}10^{\circ} 41^{\prime} 20.7^{\prime \prime} \mathrm{N} \\
63^{\circ} 52^{\prime} 29.11^{\prime \prime} \mathrm{W}\end{array}$ & $15 / 02 / 2016$ & Islote Lobos/2 & $\begin{array}{l}\text { Universidad Simón Bolivar/ by-product of the artisanal fishery of the } \\
\text { Araya Peninsula in Venezuela }\end{array}$ \\
\hline $\begin{array}{l}\text { Cape Verde- } \\
\text { Africa }\end{array}$ & $\begin{array}{l}16^{\circ} 35^{\prime} 39.4 ’ \mathrm{~N} \\
22^{\circ} 53^{\prime} 42.6^{\prime \prime} \mathrm{W}\end{array}$ & $11 / 08 / 2011$ & $\begin{array}{l}\text { Santa María, Sol } \\
\text { Island } / 8\end{array}$ & $\begin{array}{l}\text { Donation by Owen Wangensteen, Department of Animal Biology at } \\
\text { University of Barcelona. }\end{array}$ \\
\hline
\end{tabular}


Capillary electrophoresis of PCR products was performed using an Applied Biosystems 3730 DNA Analyzer at the Genomic Technologies Facility, University of Manchester (UK). Length and allele scoring of PCR products were estimated using the GeneMapper 5 (ThermoFisher Scientific) and Peakscanner v1.0 software (Applied Biosystems). Seventeen primers showed successful amplification, variable microsatellite loci, and were successfully genotyped in all 23 samples used.

\section{Data analysis}

We used the microsatellite genotypes of 23 individuals from several localities in the Caribbean and at Cape Verde (Africa) for analysis. Data were edited in Excel (Microsoft, Washington, USA) tables and converted to input files (Microsatellite Toolkit) to produce the genepop data. To detect and estimate genotyping error, data were probed using the Microchecker (Van Oosterhout, et al., 2004) software. We then tested for HardyWeinberg Equilibrium (HWE), calculated the number of alleles per loci (Na) and population, and observed heterozygosity (Ho) and expected heterozygocity (He) with the GenAlEx platform (http://biology.anu.edu.au/GenAlEx/Welcome.html). We estimated

Table 3. Characteristics of 26 nuclear microsatellite markers developed for Echinometra lucunter. F: Forward. R: Reverse. F.L: Fluorescent label. Grey: No development

\begin{tabular}{|c|c|c|c|c|c|c|c|}
\hline Reactions & Locus & & Universal tail used & Primer sequences & F.L & $\begin{array}{c}\text { Repeat } \\
\text { motif }\end{array}$ & $\begin{array}{l}\text { Allele size } \\
\text { tange (bp) }\end{array}$ \\
\hline \multirow[t]{2}{*}{ Singleplex } & \multirow[t]{2}{*}{ EL1 } & F: & GCCTCCCTCGCGCCA & AATGCTCCCCAAATTTCTCG & \multirow{2}{*}{ 6-FAM } & \multirow{2}{*}{$\mathrm{AAG} * 81$} & \\
\hline & & $\mathrm{R}:$ & & GCCTGTTACAATTTCCTGGGG & & & \\
\hline \multirow[t]{2}{*}{ Singleplex } & \multirow[t]{2}{*}{ EL2 } & F: & GCCTCCCTCGCGCCA & ATGGCTTTTGGGACAGATGG & \multirow{2}{*}{ 6-FAM } & \multirow{2}{*}{$\mathrm{AAG}^{*} 111$} & \multirow{2}{*}{$394-434$} \\
\hline & & $\mathrm{R}:$ & & TCCTCAGAAATTATGCCCGC & & & \\
\hline \multirow[t]{2}{*}{ Singleplex } & \multirow[t]{2}{*}{ EL3 } & F: & GCCTCCCTCGCGCCA & CGATGTGAGACTAGAAATGTACGG & \multirow{2}{*}{ 6-FAM } & \multirow{2}{*}{$\mathrm{AAC} * 93$} & \multirow{2}{*}{$390-407$} \\
\hline & & R: & & CGACAACTGGTACCTGGACG & & & \\
\hline \multirow[t]{2}{*}{ Singleplex } & \multirow[t]{2}{*}{ EL4 } & F: & GCCTCCCTCGCGCCA & GCATGTTCACGATTTGAAGG & \multirow{2}{*}{ 6-FAM } & \multirow{2}{*}{$\mathrm{AAG} * 90$} & \multirow{2}{*}{$241-269$} \\
\hline & & $\mathrm{R}:$ & & GCAGATGGTGGAGAAGAGG & & & \\
\hline \multirow[t]{2}{*}{ Singleplex } & \multirow[t]{2}{*}{ EL5 } & F: & GCCTCCCTCGCGCCA & CTTTCAAAGACCCGATACAACG & \multirow{2}{*}{ 6-FAM } & \multirow{2}{*}{$\mathrm{AG}^{* 90}$} & \multirow{2}{*}{$296-324$} \\
\hline & & R: & & TGCACCGAATAATGATGAGC & & & \\
\hline \multirow[t]{2}{*}{ Singleplex } & \multirow[t]{2}{*}{ EL6 } & F: & GCCTCCCTCGCGCCA & TGATCGAAACGGTGACATCC & \multirow{2}{*}{ 6-FAM } & \multirow{2}{*}{ AAAC* 104} & \multirow{2}{*}{$209-253$} \\
\hline & & $\mathrm{R}:$ & & AGTCAGATCACCGCCATGC & & & \\
\hline \multirow[t]{2}{*}{ Singleplex } & \multirow[t]{2}{*}{ EL7 } & F: & GCCTCCCTCGCGCCA & TGGGACAAAGAGAGAGCTTGG & \multirow{2}{*}{ 6-FAM } & $\mathrm{AG}^{*} 72$ & $162-171$ \\
\hline & & R: & & AGCGGATGTTGATTTACGGC & & 120 & 20 \\
\hline Multiplex1 & EL8 & F: & CAGGACCAGGCTACCGTG & GGCTTATGGTCACAGGACTGG & 6-FAM & AG*68 & \\
\hline & & R: & & GGAAGAGCTCGCTTGATTCG & & & \\
\hline & EL14 & $\mathrm{F}:$ & CACTGCTTAGAGCGATGC & TCTTCTCGATCCCTCTTTGTCC & PET & $\mathrm{AAG} * 84$ & $333-345$ \\
\hline & & R: & & TGAAGGTGCACTGATGGAGG & & & \\
\hline & EL20 & F: & CGGAGAGCCGAGAGGTG & CGCCATTAATGTCAACACCG & HEX & ATC*69 & $249-318$ \\
\hline & & R: & & AGCATCTGAATCCCCACCC & & & \\
\hline Multiplex2 & EL9 & F: & CAGGACCAGGCTACCGTG & CCGTCTTGAGAGCTATCGGC & 6-FAM & $\mathrm{AGT}^{*} 72$ & \\
\hline & & R: & & GCGTTTAAGATTCCCTTTGCC & & 250 & \\
\hline & EL15 & F: & CACTGCTTAGAGCGATGC & GTTTCACATCGGTCCGTCG & PET & $\mathrm{AAG} * 93$ & $346-400$ \\
\hline & & $\mathrm{R}:$ & & CTCCATAGCAACATGACGGG & & & \\
\hline & EL21 & F: & CGGAGAGCCGAGAGGTG & GCAGTATCATCATCGCCAGC & $\mathrm{HE}$ & & $31+3$ \\
\hline & & $\mathrm{R}:$ & & TCAGGGATTGTGTCTTTGCG & & & \\
\hline
\end{tabular}




\begin{tabular}{|c|c|c|c|c|c|c|c|}
\hline Reactions & Locus & & Universal tail used & Primer sequences & F.L & $\begin{array}{c}\text { Repeat } \\
\text { motif }\end{array}$ & $\begin{array}{l}\text { Allele size } \\
\text { tange (bp) }\end{array}$ \\
\hline \multirow[t]{6}{*}{ Multiplex3 } & \multirow[t]{2}{*}{ EL10 } & F: & CAGGACCAGGCTACCGTG & TCAGCCTAAGTGTGTTGGAAGC & \multirow{2}{*}{ 6-FAM } & \multirow{2}{*}{$\mathrm{AG}^{*} 114$} & \\
\hline & & R: & & CTCTGTGCAAAGAAGGTTAAGTGC & & & \\
\hline & \multirow[t]{2}{*}{ EL16 } & F: & CACTGCTTAGAGCGATGC & TGAAGACGTTCGTTGATTGC & \multirow{2}{*}{ PET } & \multirow{2}{*}{$\mathrm{AG} * 52$} & \multirow{2}{*}{$182-272$} \\
\hline & & R: & & GCACTTACATCAGTGGATTGC & & & \\
\hline & \multirow[t]{2}{*}{ EL22 } & $\mathrm{F}:$ & CGGAGAGCCGAGAGGTG & ACATTGAAAACACTGGGGCG & \multirow{2}{*}{ HEX } & \multirow{2}{*}{$\mathrm{AAG}^{*} 129$} & \multirow{2}{*}{$156-294$} \\
\hline & & $\mathrm{R}:$ & & ATGCATTTTCATGTGCACCC & & & \\
\hline \multirow[t]{6}{*}{ Multiplex4 } & \multirow[t]{2}{*}{ EL11 } & F: & CAGGACCAGGCTACCGTG & TGATGCTCTACATTTGAGCCC & \multirow{2}{*}{ 6-FAM } & \multirow{2}{*}{$\mathrm{AG}^{*} 68$} & \\
\hline & & R: & & TCTCCCTAGCGTTACAGGGG & & & \\
\hline & \multirow[t]{2}{*}{ EL17 } & F: & CACTGCTTAGAGCGATGC & AAGTGGGTAGGCCAACGCTACACG & \multirow{2}{*}{ PET } & \multirow{2}{*}{$\mathrm{AG} * 96$} & \multirow{2}{*}{$217-247$} \\
\hline & & $\mathrm{R}:$ & & ACGCGCATACTATAGCGTGGTGG & & & \\
\hline & \multirow[t]{2}{*}{ EL23 } & F: & CGGAGAGCCGAGAGGTG & GAGACAGAATGAAAATCGCTGC & \multirow{2}{*}{ HEX } & \multirow{2}{*}{$\mathrm{TG}^{*} 38$} & \multirow{2}{*}{$297-367$} \\
\hline & & $\mathrm{R}:$ & & CGAAGGGACTATGAAACGGG & & & \\
\hline \multirow[t]{6}{*}{ Multiplex 5} & \multirow[t]{2}{*}{ EL12 } & F: & CAGGACCAGGCTACCGTG & TGGTGCAAATGTAGCAGTCG & \multirow{2}{*}{ 6-FAM } & \multirow{2}{*}{$\mathrm{AGT}^{* 75}$} & \\
\hline & & $\mathrm{R}:$ & & CGTGGTACATTGCTTACAGCC & & & \\
\hline & \multirow[t]{2}{*}{ EL18 } & F: & CACTGCTTAGAGCGATGC & GCTCATTGGCAGATCAAACG & \multirow{2}{*}{ PET } & \multirow{2}{*}{$\mathrm{AG}^{*} 60$} & \multirow{2}{*}{$217-399$} \\
\hline & & $\mathrm{R}:$ & & CTTGCTCTCTTGTTCTATTCCCC & & & \\
\hline & EL24 & F: & CGGAGAGCCGAGAGGTG & CGGTGACCCTACAGTAAACTTGC & HFX & $\mathrm{A} G * 54$ & $300-474$ \\
\hline & & $\mathrm{R}:$ & & TCCСТCTTTCTCCCACGC & ПЕ $\lambda$ & $\mathrm{Au} \cdot 34$ & $500-4 / 4$ \\
\hline Multiplex6 & EL13 & F: & CAGGACCAGGCTACCGTG & CCATGCCCAATAATAACGCC & & & \\
\hline & & $\mathrm{R}:$ & & ATCCTTGGGCCATCTTTCC & 6-FAM & $A G^{*} 128$ & \\
\hline & EL19 & $\mathrm{F}:$ & CACTGCTTAGAGCGATGC & CAAACTAAAGACGCCCAGCC & PET & $\Delta G * \Delta \cap$ & 156.186 \\
\hline & & $\mathrm{R}:$ & & ATGAGGCTTCCCTGAATCCC & PEI & $A U^{\prime \prime} 40$ & $156-186$ \\
\hline & EL25 & F: & CGGAGAGCCGAGAGGTG & TAAACCAGGCCAGAATGACG & & & \\
\hline & & R: & & TGGGAGCTTTTAGTCCGAGC & HEX & 10.7 & \\
\hline Singleplex & EL26 & F: & CGGAGAGCCGAGAGGTG & TTCATCCAACTCTGTCACATCC & HEX & AAG*147 & \\
\hline
\end{tabular}

the Polymorphic Information Content (PIC) of each locus using the program Cervus 3.0.7 (Kalinowski, et al., 2007), and the genotypic linkage disequilibrium using GenPop (http://genepop.curtin.edu.au/). We used the Bonferroni correction method to adjust the significance levels for HWE and linkage disequilibrium tests.

\section{Results}

The Illumina MiSeq run generated 27,043,607 paired-end reads with the eight DNA samples (Honduras, Puerto Rico, and Venezuela). We obtained a further filtered list of 308 potentially amplifiable loci (PAL) with the pal_filter from the Galaxy platform and we selected 26 PAL's for screening: 14 di-, 11 tri- and one tetra-nucleotide (Table 3).

After several assays, we obtained 17 successful microsatellite markers: Nine di-, seven tri-, and one tetra nucleotide (Table 4). The allele number per locus $(\mathrm{Na})$ ranged from four to 24 while the observed (Ho) and expected $(\mathrm{He})$ heterozygosities ranged from 0.682 to 1 and 0.609 to 0.9304 , respectively.

Two out of the 17 loci deviated from HWE (EL22- present in 16 of 23 samples during microsatellite screening and EL14- present in all samples except one in San Andres Island) after Bonferroni correction for multiple testing $(\mathrm{p}<0.05)$. Homozygote excess, related to the presence of null alleles or stochasticity due to the different locality sources for one 
Table 4. Characteristics of 17 markers developed for Echinometra lucunter

\begin{tabular}{|c|c|c|c|c|c|c|c|c|c|c|c|c|}
\hline $\begin{array}{l}\text { Locus } \\
\text { name }\end{array}$ & Forward and reverse primer & Fluor & R. motif & $\begin{array}{l}\text { Locus size } \\
\text { range }\end{array}$ & $\mathbf{N}$ & $\mathbf{N a}$ & $\mathrm{Ne}$ & Ho & He & PIC & pHWE & pHWEbc \\
\hline EL2 & $\begin{array}{l}\text { ATGGCTTTTGGGACAGATGG } \\
\text { TCCTCAGAAATTATGCCCGC }\end{array}$ & $\begin{array}{c}6-F A M \\
-\end{array}$ & $(\mathrm{AAG})_{111}$ & $394-434$ & 7 & 7 & 4.900 & 0.857 & 0.796 & 0.772 & 0.817 & 1.000 \\
\hline EL3 & $\begin{array}{l}\text { CGATGTGAGACTAGAAATGTACGG } \\
\text { CGACAACTGGTACCTGGACG }\end{array}$ & $\begin{array}{c}\text { 6-FAM } \\
-\end{array}$ & $(\mathrm{AAC})_{93}$ & $390-407$ & 8 & 6 & 4.414 & 1.000 & 0.773 & 0.744 & 0.509 & 1.000 \\
\hline EL4 & $\begin{array}{l}\text { GCATGTTCACGATTTGAAGG } \\
\text { GCAGATGGTGGAGAAGAGG }\end{array}$ & $\begin{array}{c}6-F A M \\
-\end{array}$ & $(\mathrm{AAG})_{90}$ & $241-269$ & 8 & 6 & 4.000 & 1.000 & 0.750 & 0.712 & 0.559 & 1.000 \\
\hline EL5 & $\begin{array}{l}\text { CTTTCAAAGACCCGATACAACG } \\
\text { TGCACCGAATAATGATGAGC }\end{array}$ & $\begin{array}{c}\text { 6-FAM } \\
-\end{array}$ & $(\mathrm{AG})_{90}$ & 296-324 & 6 & 10 & 9.000 & 1.000 & 0.889 & 0.878 & 0.600 & 1.000 \\
\hline EL6 & $\begin{array}{l}\text { TGATCGAAACGGTGACATCC } \\
\text { AGTCAGATCACCGCCATGC }\end{array}$ & $\begin{array}{c}6-F A M \\
-\end{array}$ & $(\mathrm{AAAC})_{104}$ & $209-253$ & 8 & 4 & 2.844 & 0.875 & 0.648 & 0.592 & 0.204 & 1.000 \\
\hline EL7 & $\begin{array}{l}\text { TGGGACAAAGAGAGAGCTTGG } \\
\text { AGCGGATGTTGATTTACGGC }\end{array}$ & $\begin{array}{c}6-F A M \\
-\end{array}$ & $(\mathrm{AG})_{72}$ & $162-171$ & 8 & 4 & 2.560 & 0.875 & 0.609 & 0.530 & 0.495 & 1.000 \\
\hline EL14 & $\begin{array}{l}\text { TCTTCTCGATCCCTCTTTGTCC } \\
\text { TGAAGGTGCACTGATGGAGG }\end{array}$ & $\begin{array}{c}\text { PET } \\
-\end{array}$ & $(\mathrm{AG})_{68}$ & $333-345$ & 22 & 5 & 3.625 & 0.682 & 0.724 & 0.681 & $0.002 * *$ & $0.034 *$ \\
\hline EL15 & $\begin{array}{l}\text { GTTTCACATCGGTCCGTCG } \\
\text { CTCCATAGCAACATGACGGG }\end{array}$ & $\begin{array}{c}\text { PET } \\
-\end{array}$ & $(\mathrm{AAG})_{93}$ & $346-400$ & 20 & 16 & 10.256 & 0.800 & 0.903 & 0.895 & $0.014 *$ & 0.238 \\
\hline EL16 & $\begin{array}{l}\text { TGAAGACGTTCGTTGATTGC } \\
\text { GCACTTACATCAGTGGATTGC }\end{array}$ & $\begin{array}{c}\text { PET } \\
-\end{array}$ & $(A G)_{52}$ & $182-272$ & 16 & 19 & 15.059 & 0.875 & 0.934 & 0.930 & 0.144 & 1.000 \\
\hline EL17 & $\begin{array}{l}\text { AAGTGGGTAGGCCAACGCTACACG } \\
\text { ACGCGCATACTATAGCGTGGTGG }\end{array}$ & $\begin{array}{c}\text { PET } \\
-\end{array}$ & $(\mathrm{AG})_{96}$ & $217-247$ & 19 & 13 & 8.022 & 0.789 & 0.875 & 0.864 & 0.590 & 1.000 \\
\hline EL18 & $\begin{array}{l}\text { GCTCATTGGCAGATCAAACG } \\
\text { CTTGCTCTCTTGTTCTATTCCCC }\end{array}$ & $\begin{array}{c}\text { PET } \\
-\end{array}$ & $(\mathrm{AG})_{60}$ & 217-399 & 21 & 22 & 11.919 & 0.810 & 0.916 & 0.911 & $0.024 *$ & 0.408 \\
\hline EL19 & $\begin{array}{l}\text { CAAACTAAAGACGCCCAGCC } \\
\text { ATGAGGCTTCCCTGAATCCC }\end{array}$ & $\begin{array}{c}\text { PET } \\
-\end{array}$ & $(\mathrm{AG})_{40}$ & $156-186$ & 11 & 10 & 5.261 & 0.909 & 0.810 & 0.792 & 0.275 & 1.000 \\
\hline EL20 & $\begin{array}{l}\text { CGCCATTAATGTCAACACCG } \\
\text { AGCATCTGAATCCCCACCC }\end{array}$ & $\begin{array}{c}\text { HEX } \\
-\end{array}$ & $(\mathrm{ATC})_{69}$ & $249-318$ & 20 & 16 & 10.000 & 0.850 & 0.900 & 0.892 & $0.021 *$ & 0.357 \\
\hline EL21 & $\begin{array}{l}\text { GCAGTATCATCATCGCCAGC } \\
\text { TCAGGGATTGTGTCTTTGCG }\end{array}$ & $\begin{array}{c}\text { HEX } \\
-\end{array}$ & $(\text { ATC })_{126}$ & $315-561$ & 22 & 24 & 13.444 & 1.000 & 0.926 & 0.921 & 0.229 & 1.000 \\
\hline EL22 & $\begin{array}{l}\text { ACATTGAAAACACTGGGGCG } \\
\text { ATGCATTTTCATGTGCACCC }\end{array}$ & $\begin{array}{c}\text { HEX } \\
-\end{array}$ & $(\mathrm{AAG})_{129}$ & $156-294$ & 15 & 13 & 9.783 & 0.733 & 0.898 & 0.889 & $0.001 * * *$ & $0.017^{*}$ \\
\hline EL23 & $\begin{array}{l}\text { GAGACAGAATGAAAATCGCTGC } \\
\text { CGAAGGGACTATGAAACGGG }\end{array}$ & $\begin{array}{c}\text { HEX } \\
-\end{array}$ & $(\mathrm{TG})_{38}$ & $297-367$ & 19 & 18 & 11.108 & 0.842 & 0.910 & 0.904 & $0.017^{*}$ & 0.289 \\
\hline EL24 & $\begin{array}{l}\text { CGGTGACCCTACAGTAAACTTGC } \\
\text { TCССТCTTTCTCCCACGC }\end{array}$ & $\begin{array}{c}\text { HEX } \\
-\end{array}$ & $(\mathrm{AG})_{54}$ & $300-474$ & 10 & 13 & 10.000 & 0.800 & 0.900 & 0.892 & 0.130 & 1.000 \\
\hline
\end{tabular}

Fluor: Fluorescent dye, R. motif: Motif of repetition, N: Sample size, Na: Allele number, Ne: Allele effective number, Ho: Observed heterozygosity, He: Expected heterozygosity, PIC: Polymorphic information content, pHWE: Hardy-Weingberg Equilibrium, pHWEbc: Hardy-Weingberg Equilibrium with Bonferroni Correction method. *: $\mathrm{p}<0,05, * *: \mathrm{p}<0,01 ; * * * \mathrm{p}<0,001$

population, might explain these deviations. Polymorphic information content (PIC) ranging from 0.530 to 0.930 shows normal polymorphism behavior (Table 4). We did not detect linkage disequilibrium between any locus pair after the Bonferroni correction, which means that in general, all the loci were independent.

\section{Discussion}

Microsatellite development is useful for a variety of genetic analyses of population structure. In this case, these markers will be used for the first time to confirm the influence of marine barriers to genetic flow among populations of $E$. lucunter lucunter throughout the Caribbean Sea. 
For many years, based upon the biological characteristics of marine organisms and barriers such as mouths of rivers, marine currents, physicochemical parameters, and others in the Caribbean Sea, several authors have wondered if the organisms of this area are genetically homogeneous or segregated. While few marine barriers have been identified (Carlin, et al., 2003, Baums, et al., 2005, Cowen, et al., 2006, Taylor \& Hellberg, 2003, 2006), several biophysical models have been proposed to identify patterns of connectivity and potential barriers to larval dispersal of reef fish (Schultz \& Cowen, 1994; Paris \& Cowen, 2004; Cowen, et al., 2006). There are few genetic evaluations of other marine groups providing evidence of both connectivity and phylogeographic breaks for the Caribbean (Avise, 1992; Carlin, et al., 2003; Taylor \& Hellberg, 2003; Baums, et al., 2005).

According to Fox, et al. (2019), ecological and conservation studies often focus upon non-model species for which genetic markers are not available, and Echinometra species are an example. The methodology used to isolate and characterize the E. lucunter loci followed the method of multi-individual microsatellite identification (MiMi) proposed by Fox, et al. (2019) with the combination of affordable Next Generation Sequencing (NGS) and freely available bioinformatics tools. Our positive results support once again the effectiveness and speed of this method.

The application of the microsatellite markers developed herein to additional $E$. lucunter populations will allow understanding how the genetic diversity is distributed in this benthic species throughout the Caribbean Sea considering its biological features and the marine barriers described for the region.

\section{Conclusions}

Seventeen (17) new microsatellites were developed and characterized for the sea urchin Echinometra lucunter lucunter. The markers fill up the gap of information regarding tools to characterize the genetic structure of this species in the Caribbean Sea. Due to the absence of microsatellites within the species of the genus Echinometra, it was necessary to develop and characterize a number of this kind of markers to determine the genetic structure of Echinometra lucunter lucunter in the Caribbean Sea. The successful isolation and characterization of these microsatellites will contribute to genetic and marine connectivity studies of Echinometra lucunter lucunter and Echinometra lucunter polypora through their broad range of distribution in the Atlantic Ocean. Information on population genetics of this species is crucial for the assessment of the ecological situation of their populations and the development of management strategies when necessary.

\section{Acknowledgments}

Special thanks are given to Dra. Sarah Griffiths, Manchester Metropolitan University, for her support on microsatellite development and all the work related with the molecular laboratory and the correct use of Bioinformatic Tool Galaxy. Thanks to Rachell E. Antwis for sequencing work at Salford University. Numerous people and institutions helped with sample collection and logistics throughout the Caribbean - Cape Verde (Africa), as well as with permits: Mayra Núñez (Instituto Nacional de Conservación y Desarrollo Forestal, Áreas Protegidas y Vida Silvestre, Honduras: Permit_Resolution-DE-MP-080-2015), Ricardo Betancur (Universidad de Puerto Rico, Rio Piedras Campus, Departamento de Recursos Naturales y Ambientales: DRNA 2016-IC-052), Nikolaos Schizas and Mónica Alfaro (Universidad de Puerto Rico, Mayagüez Campus), Ana Carolina Peralta (Universidad Simón Bolivar, Venezuela), Owen Wangensteen (Universitetet I Troms, Norway), Adriana Osorno (INVEMAR, Colombia), Ministry of Environment and Sustainable Development (Colombia- National Authority of Environmental Licenses (ANLA): Resolution number 255, 12 of March of 2014 and Export permit No. 00848). Thanks to Gilberto Orozco at Universidad Nacional de Colombia for his help with analysis data. This research was supported by Colciencias, fellowship call 267-2012 (PhD grant) 
and research grant No. 116171450647; UNAL internal financial help; the Ecology and Environment Research Centre-EERC at MMU (grant R60010-sampling, laboratory and analysis in Manchester); Corporation Center of Excellence in Marine Sciences - CEMarin (Call \# 12 of 2018), and contribution No. 486 of Instituto de Estudios en Ciencias del Mar, CECIMAR, Universidad Nacional de Colombia.

\section{Author contributions}

MBS was the person in charge of the results and their respective analyses, and she wrote and sent the manuscript to the journal. YFC worked with the principal author in the obtention of results and their respective analysis. LMB directly advised the work and supported the sample collection and laboratory processes, and participated in the manuscript writing. GF collaborated directly to laboratory results and actively participated in the edition of the manuscript. TDH collaborated in the laboratory work. NHCC advised the manuscript writing, and RP participated in the editing of the document.

\section{Conflict of interests}

We have no conflicts of interest to report.

\section{References}

Avise, J. C. (1992). Molecular population structure and biogeographic history of a regional fauna a case history with lessons for conservative biology. Oikos. 63: 62-76. Doi: 10.2307/3545516

Baums, I. B., Miller, M. W., Hellberg, M. E. (2005). Regionally isolated populations of an imperiled Caribbean coral, Acropora palmata. Mol Ecol. 14: 1377-1390. Doi: 10.1111/j.1365294X.2005.02489.x

Blacket, M. J., Robin. C., Good, R. T, Lee, S. F, Miller, A. D. (2012). Universal primers for fluorescent labelling of PCR fragments - an effective approach to genotyping by fluorescence. Mol Ecol Resour. 1: 456-463. Doi: 10.1111/j.1755-0998.2011.03104.x

Carlin, J. L., Robertson, D. R., Bowen, B. W. (2003). Ancient divergences and recent connections in two tropical Atlantic reef fishes Epinephelus adscensionis and Rypticus saponaceous (Percoidei: Serranidae). Mar Biol. 143: 1057-1069. Doi: 10.1007/s00227-003-1151-3

Cowen, R. K., Paris, C. B., Srinivasan, A. (2006). Scaling of population connectivity in marine populations. Sci. 311: 522-527. Doi: 10.1126/science.1122039

Culley, T. M., Stamper, T. I., Stokes, R. L., Brzski, J. R., Hardman, N. A., Klooster, M. R, Merritt, B. J. (2013). An efficient technique for primer development and application that integrates fluorescent labelling and multiplex PCR. Appl Plant Sci. 1 (10): 1-10. Doi: 10.3732/apps. 1300027

Fox, G., Preziosi, R. F., Antwis, R. E., Benavides-Serrato M., Combe, F. J., Harris, W. E., Hartley, I. R., Kitchener, A. C., De Kort, S. R., Nekaris, A. I., Rowntree, J. K. (2019). Multi-individual microsatellite identification: A multiple genome approach to microsatellite design (MiMi). Mol Ecol Resour. 19 (6): 1-9. Doi: 10.1111/1755-0998.13065

Griffiths, S. M., Fox, G., Briggs, P. J., Donaldson, I. J., Hood, S., Richardson, P., Leaver, G. W., N. K. Truelove, Preziosi, R. F. (2016). A Galaxy-based bioinformatics pipeline for optimised, streamlined microsatellite development from Illumina next-generation sequencing data. Conserv Genet Resour. 8: 481-486. Doi: 10.1007/s12686-016-0570-7

Kalinowski, S. T., Taper, M. L., Marshall, T.C. (2007). Revising how the computer program CERVUS accommodates genotyping error increases success in paternity assignment. Mol Ecol. 16: 1099-1106. Doi: 10. 1111/j.1365-294X.2007.03089.x

McCartney, M. A., Keller, G., Lessios, H. A. (2000). Dispersal barriers in tropical oceans and speciation in Atlantic and eastern Pacific sea urchins of the genus Echinometra. Mol. Ecol. 9: 1391-1400. Doi: 10.1046/j.1365-294x.2000.01022.x

Paris, C. B., Cowen, R. K. (2004). Direct evidence of a biophysical retention mechanism for coral reef fish larvae. Limnol. 49 (6): 1964-1979. Doi: 10.4319/1o.2004.49.6.1964

Pawson, D. L. (1978). The echinoderm fauna of Ascension Island, South Atlantic Ocean. Smithsonian Contrib. Mar. Sci, 2: 1-29.

Qiagen. DNeasy ${ }^{\circledR}$ Blood \& Tissue Handbook [pdf] (2006). Date of entry: January 15, 2015. Available in: http://mvz.berkeley.edu/egl/inserts/DNeasy_Blood_\&_Tissue_Handbook.pdf 
Qiagen. Type-it Microsatellite PCR Handbook [pdf] (2009). Date of entry: March 14, 2015. Available in: http://www.qiagen.com/Products/Catalog/Assay-Technologies/End-Point-PCR-and-RTPCR-Reagents/Type-it-Microsatellite-PCR-Kit\#resources

Schultz, E. T. \& Cowen, R. K. (1994). Recruitment of coral-reef fishes to Bermuda - local retention or long-distance transport. Mar Ecol Prog Ser. 109: 15-28. Doi: 10.3354/meps 111015

Selkoe, K. A. \& Toonen, R. J. (2006). Microsatellites for ecologists: A practical guide to using and evaluating microsatellite markers. Ecol Lett. 9: 615-29. Doi: 10.1111/j.1461-0248.2006.00889.x

Sunnucks, P. (2000). Efficient genetic markers for population biology. Trends Ecol Evol. 15 (5): 199-203. Doi: 10.1016/S0169-5347(00)01825-5

Taylor, M. S. \& Hellberg, M. E. (2003). Genetic evidence for local retention of pelagic larvae in a Caribbean reef fish. Sci. 299: 107-109. Doi: 10.1126/science.1079365

Taylor, M. S. \& Hellberg, M. E. (2006). Comparative phylogeography in a genus of coral reef fishes: biogeographic and genetic concordance in the Caribbean. Mol Ecol. 15: 695-707. Doi: 10.1111/j.1365-294X.2006.02820.x

Van Oosterhout, C., Hutchison, W. F., Shipley, P., Wills, D. P. M. (2004). Micro-Checker: Software for identifying and correcting genotyping errors in microsatellite data. Mol. Ecol. Notes. 4: 535-538. Doi: 10.1111/j.1471-8286.2004.00684.x 